\title{
Multi-spin counter-diabatic driving in many-body quantum Otto refrigerators
}

\author{
Andreas Hartmann ${ }^{1}$, Victor Mukherjee ${ }^{2}$, Glen Bigan Mbeng ${ }^{1}$, Wolfgang Niedenzu ${ }^{1}$, and \\ Wolfgang Lechner ${ }^{1}$ \\ ${ }^{1}$ Institut für Theoretische Physik, Universität Innsbruck, Technikerstraße 21a, A-6020 Innsbruck, Austria \\ ${ }^{2}$ Department of Physical Sciences, IISER Berhampur, Berhampur 760010, India
}

\section{Quantum refrigerators pump heat from} a cold to a hot reservoir. In the few-particle regime, counter-diabatic (CD) driving of, originally adiabatic, workexchange strokes is a promising candidate to overcome the bottleneck of vanishing cooling power. Here, we present a finite-time many-body quantum refrigerator that yields finite cooling power at high coefficient of performance, that considerably outperforms its non-adiabatic counterpart. We employ multi-spin CD driving and numerically investigate the scaling behavior of the refrigeration performance with system size. We further prove that optimal refrigeration via the exact $C D$ protocol is a catalytic process.

December 21, 2020

\section{Introduction}

Heat engines and refrigerators are a cornerstone of modern physics and indispensable in today's society [1]. Unravelling their fundamental laws in the few-particle regime has lead to the study of so-called quantum heat engines and refrigerators [2-9]. With the recent progress in controling quantum systems [11-13], such quantum heat engines could already be experimentally realized using various single-body quantum working media (WM) [14-19]. Whereas heat engines convert thermal energy into work, their counterparts, namely refrigerators, cool down a cold bath by pumping heat from the cold to the hot reservoir, thereby consuming work [7, 8, 20-25]. The maximum coefficient of performance $(\mathrm{CoP})$ of refrigerators is limited by the Carnot CoP [26]. For Andreas Hartmann: andreas.Hartmann@uibk.ac.at Glen Bigan Mbeng: glen.mbeng@uibk.ac.at these infinitely long (adiabatic) cycle times, the corresponding cooling power naturally converges to zero. A natural question thus arises whether such quantum refrigerators can be driven in finite time, yet produce a finite cooling power.

So called shortcuts to adiabaticity (STA) [2730] are a promising candidate to overcome this fundamental bottleneck, due to minimizing quantum friction [31-33] during the work-exchange strokes. These STA methods [34-39] including counter-diabatic (CD) driving [39-44] where an additional CD Hamiltonian is applied to suppress any transitions between the system's eigenstates during the Hamiltonian's dynamics, have recently been applied in the field of quantum thermodynamics to enhance the performance of quantum heat engines [45-49] and refrigerators [50, 51] using single-body quantum WM. For the latter, exact local CD terms can be found analytically [36, 38].

In general, identifying the exact $\mathrm{CD}$ term requires a priori knowledge of the system's eigenstates at all times during the Hamiltonian's dynamics and which is numerically and experimentally impracticable for many-body WM. With this challenge in mind, Sels and Polkovnikov [42] have developed a variational principle where approximate multi-spin CD terms can be found. Based on this method, a quantum heat engine using a many-body quantum WM and local 1-spin CD Hamiltonian could be implemented [52]. However, the latter are poor approximations and can only efficiently speed up an Otto cycle with weakly coupled WM. The question whether a cycle with WM with complex all-to-all connectivity - that provides versatile applications in state-ofthe-art experimental setups in the field of quantum computation and simulation, cf. Refs. [5356 - can be sped-up is thus still open. 
In this work, we present a finite-time manybody quantum refrigerator (QR) with finite cooling power using additional approximate multispin $\mathrm{CD}$ terms. The WM is described by an all-toall connected Ising spin system and can efficiently be driven using non-local, yet well approximated $p$-spin CD terms. We numerically demonstrate a large enhancement in cooling power and CoP along with improved scaling behaviour of the cooling power with the system size for the sped-up QR compared to its original non-adiabatic and traditional 1-spin CD counterparts. For the QR with single-body quantum WM, we find an analytical expression for the CoP. For the many-body WM, we provide an analytical proof that exact CD driving implies a zero work component of the additional external control device and thus is a catalytic process. Remarkably, the latter fully assists the piston to run the QR in this case, mimicking the adiabatic quantum cycle, yet in finite time.

This work is structured as follows: In Sec. 2, we introduce the quantum Otto refrigerator using a many-body spin system as its quantum WM and present multi-spin local CD driving. In Sec. 3, we numerically analyze the performance of the corresponding refrigerators and conclude our results in Sec. 4 while giving an outlook on future research.

\section{Methods}

\subsection{Quantum Otto refrigerator}

Quantum Otto refrigerators cyclically pump heat from a cold to a hot reservoir by consuming work. Its corresponding four-stroke quantum Otto cycle [8] consists of two heat-exchange strokes where the quantum WM is alternatingly coupled to two heat baths - and two work-exchange strokes.

The four strokes are (cf. Fig. 1):

1. Adiabatic compression $(A \rightarrow B)$ : Initially, at cold temperature $T_{\mathrm{c}}=1 / \beta_{\mathrm{c}}$, Hamiltonian $H_{0}\left(\lambda_{\mathrm{c}}\right)$ with working parameter $\lambda_{\mathrm{c}}:=\lambda(t=0)$ and in the thermal state $\rho_{A}=e^{-H_{0}\left(\lambda_{\mathrm{c}}\right) / T_{\mathrm{c}}} / \operatorname{Tr}\left[e^{-H_{0}\left(\lambda_{\mathrm{c}}\right) / T_{\mathrm{c}}}\right]$, the $\mathrm{WM}$ is changed adiabatically during the first isentropic stroke of duration $\tau_{1}$ with final Hamiltonian $H_{0}\left(\lambda_{\mathrm{h}}\right)$ and mixed state $\rho_{B}$ with $\lambda_{\mathrm{h}}:=$ $\lambda(t=\tau)$.

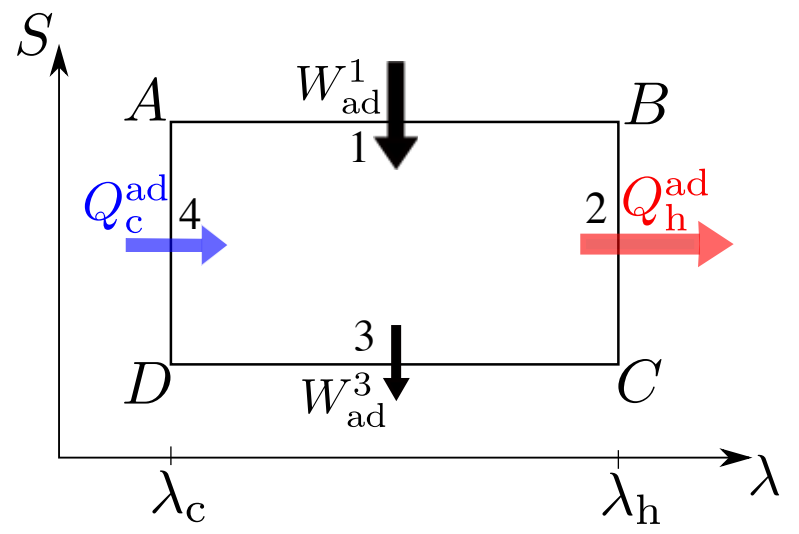

Figure 1: Entropy-working parameter diagram of quantum Otto refrigerator. The quantum Otto cycle consists of two adiabatic ( 1 and 3 ) and two thermal strokes (2 and 4$)$. At the end of the cycle, the heat $Q_{\mathrm{c}}^{\mathrm{ad}}$ is pumped from the cold bath at temperature $T_{\mathrm{c}}$ into the hot bath at temperature $T_{\mathrm{h}}$ by consuming the work $W_{\text {ad }}^{1}+W_{\text {ad }}^{3}>0$.

2. Hot isochore $(B \rightarrow C)$ : The WM is brought in contact with the hot thermal bath at temperature $T_{\mathrm{h}}$ until it equilibrates to the thermal state $\rho_{C}=e^{-H_{0}\left(\lambda_{\mathrm{h}}\right) / T_{\mathrm{h}}} / \operatorname{Tr}\left[e^{-H_{0}\left(\lambda_{\mathrm{h}}\right) / T_{\mathrm{h}}}\right]$. During this heat-exchange stroke of duration $\tau_{2}$, the heat $Q_{\mathrm{h}}$ is imparted to the hot bath and the WM's Hamiltonian $H_{0}\left(\lambda_{\mathrm{h}}\right)$ remains unchanged.

3. Adiabatic expansion $(C \rightarrow D)$ : The Hamiltonian $H_{0}\left(\lambda_{\mathrm{h}}\right)$ changes back to $H_{0}\left(\lambda_{\mathrm{c}}\right)$ during the stroke duration $\tau_{3}$ until the WM attains the state $\rho_{D}$.

4. Cold isochore $(D \rightarrow A)$ : The WM is brought in contact with the cold bath at temperature $T_{\mathrm{c}}$ and working parameter $\lambda_{\mathrm{c}}$ and cools down during the duration $\tau_{4}$ to its originally initial thermal state $\rho_{A}$.

During one cycle, the WM extracts the heat $Q_{\mathrm{c}}^{\text {ad }}:=\left\langle H_{0}\left(\lambda_{\mathrm{c}}\right)\right\rangle_{\rho_{A}}-\left\langle H_{0}\left(\lambda_{\mathrm{c}}\right)\right\rangle_{\rho_{D}}>0$ from the cold thermal bath, thereby consumes the work $W_{\text {ad }}^{1+3}:=W_{\text {ad }}^{1}+W_{\text {ad }}^{3}>0$ (here, $W_{\mathrm{ad}}^{1}=\left\langle H_{0}\left(\lambda_{\mathrm{h}}\right)\right\rangle_{\rho_{B}}-\left\langle H_{0}\left(\lambda_{\mathrm{c}}\right)\right\rangle_{\rho_{A}}$ and $W_{\mathrm{ad}}^{3}=$ $\left.\left\langle H_{0}\left(\lambda_{\mathrm{c}}\right)\right\rangle_{\rho_{D}}-\left\langle H_{0}\left(\lambda_{\mathrm{h}}\right)\right\rangle_{\rho_{C}}\right)$. A positive (negative) sign of the work components corresponds to work performed on (extracted from) the WM. Analogously, a positive (negative) sign of the heat corresponds to heat extracted from (imparted to) the thermal bath.

The cooling power is defined as the pumped heat $Q_{\mathrm{c}}^{\text {ad }}$ over the total cycle time $\tau_{\text {cycle }}=\sum_{l=1}^{4} \tau_{l}$, 
i.e.,

$$
\mathcal{J}_{\text {ad }}=\frac{\text { pumped heat }}{\text { cycle duration }}=\frac{Q_{\mathrm{c}}^{\text {ad }}}{\tau_{\text {cycle }}} .
$$

The CoP of the Otto cycle is defined as the heat $Q_{\mathrm{c}}^{\text {ad }}$ pumped from the cold bath over the consumed work $W_{\text {ad }}^{1}+W_{\text {ad }}^{3}$, i.e.,

$$
\epsilon_{\mathrm{ad}}=\frac{\text { pumped heat }}{\text { consumed work }}=\frac{Q_{\mathrm{c}}^{\mathrm{ad}}}{W_{\mathrm{ad}}^{1}+W_{\mathrm{ad}}^{3}} .
$$

In the adiabatic limit - where the isentropic strokes with durations $\tau_{1}$ and $\tau_{3}$ are infinitely long - the cooling power of these adiabatic quantum refrigerators goes to zero, i.e., $\lim _{\tau_{1}, \tau_{3} \rightarrow \infty} \mathcal{J}_{\text {ad }} \rightarrow 0$. To overcome this bottleneck, one can implement so called STA techniques for the two work-exchange strokes.

\subsection{Quantum working medium}

As our quantum WM, we consider an all-to-all connected Ising spin chain with Hamiltonian

$$
\begin{aligned}
H_{0}(t) & =-[1-\vartheta(t)] \sum_{j=1}^{N} h_{j} \sigma_{j}^{x} \\
& -\vartheta(t)\left[\sum_{j=1}^{N} b_{j} \sigma_{j}^{z}+\sum_{j=1}^{N} \sum_{k<j} J_{j k} \sigma_{j}^{z} \sigma_{k}^{z}\right]
\end{aligned}
$$

where $h_{j}$ and $b_{j}$, respectively, are the timedependent transverse and longitudinal magnetic field strengths at site $j$ and $J_{j k}$ the interaction strength between spins at sites $j$ and $k . \vartheta(t)$ is a continuous function that fulfills $\vartheta(t=0)=0$ and $\vartheta\left(t=\tau_{1}\right)=1$. For the second isentropic stroke the initial and final values of the function $\vartheta(t)$ are interchanged.

Throughout this work, we parametrize the working parameters $\lambda_{\mathrm{c}}$ and $\lambda_{\mathrm{h}}$ with the magnetic fields and interaction strengths at each point of Fig. 1, i.e. $\lambda_{c}:=\left\{h_{j, \mathrm{i}}, b_{j, \mathrm{i}}, J_{j k, \mathrm{i}}\right\}$ and $\lambda_{\mathrm{h}}:=\left\{h_{j, \mathrm{f}}, b_{j, \mathrm{f}}, J_{j k, \mathrm{f}}\right\}$. Here, $h_{j, \mathrm{i}}, b_{j, \mathrm{i}}$ and $J_{j k, \mathrm{i}}$ are the values of the magnetic fields and interaction strengths at points $A$ and $D$, and $h_{j, \mathrm{f}}, b_{j, \mathrm{f}}$ and $J_{j k, \mathrm{f}}$ at points $B$ and $C$, respectively. The explicit forms of the functions $h_{j}(t), b_{j}(t)$ and $J_{j k}(t)$ as well as the sweep function $\vartheta(t)$ are given in Appendix A.

\subsection{Multi-spin counter-diabatic driving}

The underlying idea of CD driving [39-44] is to efficiently drive an adiabatic evolution of a Hamiltonian in finite time by suppressing transitions between its eigenstates. Thus, we always track the instantaneous eigenstates during the whole sweep. Finding the exact CD term requires a priori knowledge of the system's eigenstates for every time during the sweep which is numerically and experimentally challenging.

In order to overcome this bottleneck, an analytical variational principle has been developed recently $[42,43]$ to find approximate CD terms. In this work, we drive the WM during the isentropic strokes with the total Hamiltonian

$$
H_{\mathrm{STA}}(t)=H_{0}(t)+H_{\mathrm{CD}}(t)
$$

where $H_{0}(t)$, Eq. (3), is the original non-adiabatic and $H_{\mathrm{CD}}(t)$ the additional CD Hamiltonian that suppresses coherences in the WM that cause quantum friction in finite-time sweeps [31-33].

The additional CD Hamiltonian reads

$$
H_{\mathrm{CD}}(t)=\dot{\vartheta}(t) \mathcal{A}_{\vartheta}(t)
$$

with $\mathcal{A}_{\vartheta}(t)$ the exact adiabatic gauge potential $[42,43,57]$ and $\dot{\vartheta}(t)$ the derivative of the sweep function of Eq. (3). We rely on an approximate adiabatic gauge potential $\mathcal{A}_{\vartheta}^{*}$ that contains $p$-spin terms (with $p \leq N$ ) and an odd number of $\sigma^{y}$ terms (e.g., $\sigma_{j}^{y}$ for $p=1, \sigma_{j}^{y}, \sigma_{j}^{y} \sigma_{k}^{x}$ and $\sigma_{j}^{y} \sigma_{k}^{z}$ for $p=2$, etc.). For $p=N$, we obtain the solution of the exact adiabatic gauge potential that entails all combinations of $N$-spin terms (cf. Ref. [58] in the case of quantum criticality). As an example, for $p=1$ we apply the ansatz $\mathcal{A}_{\vartheta}^{*}=\sum_{j=1}^{N} \alpha_{j} \sigma_{j}^{y}$ and solve for the optimal solution of each coefficient $\alpha_{j}$ by minimizing the operator distance between the exact and approximate adiabatic gauge potential. For more details, see Appendix B and Refs. [42, 43, 57].

We note, that we apply the CD Hamiltonian only in the isentropic strokes as these are normally much longer than the thermalization strokes. However, techniques to speed up the latter have been also developed recently [48, 59-62].

\subsection{Quantum refrigerator under STA}

The introduction of the additional approximate $\mathrm{CD}$ term $H_{\mathrm{CD}}^{*}(t)$ in the Hamiltonian's dynamics during the, originally non-adiabatic, workexchange strokes requires a careful definition of work, cooling power and CoP. In Ref. [52], the division

$$
W_{\mathrm{STA}}^{l}=W_{0}^{l}+W_{\mathrm{CD}}^{l}
$$


with $W_{\mathrm{STA}}^{l} \equiv \Delta E=\int_{0}^{\tau_{l}} \operatorname{Tr}\left[\rho(t) \dot{H}_{\mathrm{STA}}(t)\right] d t$ the total exchanged work for each of the two workexchange strokes $l \in\{1,3\}$ with duration $\tau_{l}$ has been introduced. The corresponding work components thus read

$$
\begin{aligned}
W_{0}^{l} & =\int_{0}^{\tau_{l}} \operatorname{Tr}\left[\rho(t) \dot{H}_{0}(t)\right] d t, \\
W_{\mathrm{CD}}^{l} & =\int_{0}^{\tau_{l}} \operatorname{Tr}\left[\rho(t) \dot{H}_{\mathrm{CD}}(t)\right] d t
\end{aligned}
$$

where the work $W_{0}^{l}$ stems from the piston and $W_{\mathrm{CD}}^{l}$ from the external control device that implements $H_{\mathrm{CD}}^{*}(t)$.

As we will show in more detail in Appendix C, this work component $W_{\mathrm{CD}}^{l}$ is zero if the additional adiabatic gauge potential and thus the CD Hamiltonian $H_{\mathrm{CD}}^{*}(t)$ is exact, i.e., $\mathcal{A}_{\vartheta}^{*}(t)=\mathcal{A}_{\vartheta}(t)$ and thus $H_{\mathrm{CD}}^{*}(t)=H_{\mathrm{CD}}(t)$. Hence, the work component $W_{\mathrm{CD}}^{1+3}$ stemming from the external control device during one cycle can be seen as an artifact of inexact, e.g. 1-spin CD driving (cf. Ref. [52]). Experimentally it is advantageous to have a vanishing contribution from the external control device as we want the external control device to fully assist the piston instead of just exploiting it to run the quantum refrigerator. In other words, the exact $\mathrm{CD}$ drive is catalytic in the sense that it allows for speeding up the cycle without the external control device being altered (charged or discharged) after a cycle. Therefore, as we will see later, applying multi-spin CD terms can efficiently speed up the cycle without excessively exploiting the external control device.

The cooling power under STA is given by

$$
\mathcal{J}_{\mathrm{STA}}:=\frac{\text { pumped heat }}{\text { cycle duration }}=\frac{Q_{\mathrm{c}}}{\tau_{\text {cycle }}}
$$

and the CoP reads

$$
\epsilon_{\mathrm{STA}}:=\frac{\text { pumped heat }}{\text { consumed work }}=\frac{Q_{\mathrm{c}}}{W_{\mathrm{STA}}^{1}+W_{\mathrm{STA}}^{3}}
$$

where $W_{\mathrm{STA}}^{1+3}=W_{\mathrm{STA}}^{1}+W_{\mathrm{STA}}^{3}>0$ is the total work performed on (consumed by) the WM per cycle. Note the difference between the pumped heat $Q_{\mathrm{c}}^{\text {ad }}$ for the adiabatic [cf. Eqs. (1) and (2)] and $Q_{\mathrm{c}}$ for the sped-up cycle [cf. Eqs. (9) and (10)].

For the case of a single-body quantum WM that is modelled by Eq. (3) with $N=1$, i.e., this reduces to the Landau-Zener (LZ) model, the
CoP evaluates to

$$
\epsilon_{\mathrm{LZ}}:=\frac{h_{\mathrm{x}, \mathrm{i}}}{b_{\mathrm{z}, \mathrm{f}}-h_{\mathrm{x}, \mathrm{i}}}
$$

where $h_{\mathrm{x}, \mathrm{i}}$ is the initial value of the transverse magnetic field in the first isentropic stroke and $b_{z, \mathrm{f}}$ the final value of the longitudinal magnetic field strength, respectively. This expression is positive provided that $b_{\mathrm{z}, \mathrm{f}}>h_{\mathrm{x}, \mathrm{i}}$ and is limited by the Carnot CoP $\epsilon_{\mathrm{C}}=T_{\mathrm{c}} /\left(T_{\mathrm{h}}-T_{\mathrm{c}}\right)$ [26] (see Appendix D for more details).

\section{Numerical Results}

In this section, we present the numerical results of the proposed quantum Otto refrigerator. To this end, we used the QuTip 4.2 [63] Python package to simulate the quantum Otto cycle with (i) nonadiabatic $\left[H_{0}(t)\right.$, Eq. (3)] and (ii) STA Hamiltonian $\left[H_{\mathrm{STA}}^{*}(t)\right.$, Eq. (4)] with $p$-spin CD terms $\left[H_{\mathrm{CD}}^{*}(t)\right.$, Eq. (5)]. We numerically solved the von Neumann equation for the isentropic strokes and computed the heat $Q_{\mathrm{c}}$ and $Q_{\mathrm{h}}$ for the two thermalization strokes as the energy difference between points $B$ and $C$ as well as $D$ and $A$ (cf. Fig. 1), respectively. Throughout this numerical performance analysis, the temperatures for the cold and hot bath are set to $T_{\mathrm{c}}=0.2$ and $T_{\mathrm{h}}=0.4$, respectively. The values for the working parameters at points $A$ and $D$ as well as $B$ and $C$ in Fig. $1 \mathrm{read} \lambda_{\mathrm{c}}=\left\{h_{j, \mathrm{i}}=0.2, b_{j, \mathrm{i}}=0, J_{j k, \mathrm{i}}=0\right\}$ and $\lambda_{\mathrm{h}}=\left\{h_{j, \mathrm{f}}=0, b_{j, \mathrm{f}}=0.5, J_{j k, \mathrm{f}}=0.1\right\}$, respectively, for both, non-adiabatic $H_{0}(t)$ and STA Hamiltonian $H_{\mathrm{STA}}^{*}(t)$ with $p$-spin CD Hamiltonian $H_{\mathrm{CD}}^{*}(t)$ for different $p$. At each point $A$, $B, C$ and $D$, the additionally applied CD Hamiltonian is zero, i.e., $H_{\mathrm{CD}}^{*}\left(t=\sum_{j} \tau_{j}\right)=0$ where $j \in\{0,1,2,3\}$ and $\tau_{0}=0$. The durations $\tau_{2}$ and $\tau_{4}$ of each thermalization stroke are set to 0.1 .

\subsection{Scaling with system size}

Our primary goal is to speed up the quantum refrigerator, i.e., we want to pump as much heat $Q_{\mathrm{c}}$ as possible from the cold to the hot reservoir in the shortest amount of time. Thus, we are particularly interested in the cooling power $\mathcal{J}$ during one cycle and its scaling behaviour for different system sizes $N$.

Figure 2 depicts the cooling power $\mathcal{J}$ for (i) non-adiabatic $\left[H_{0}(t)\right.$, Eq. (3)] and (ii) STA Hamiltonian $\left[H_{\mathrm{STA}}^{*}\right.$, Eq. (4)] for different $p$-spin 


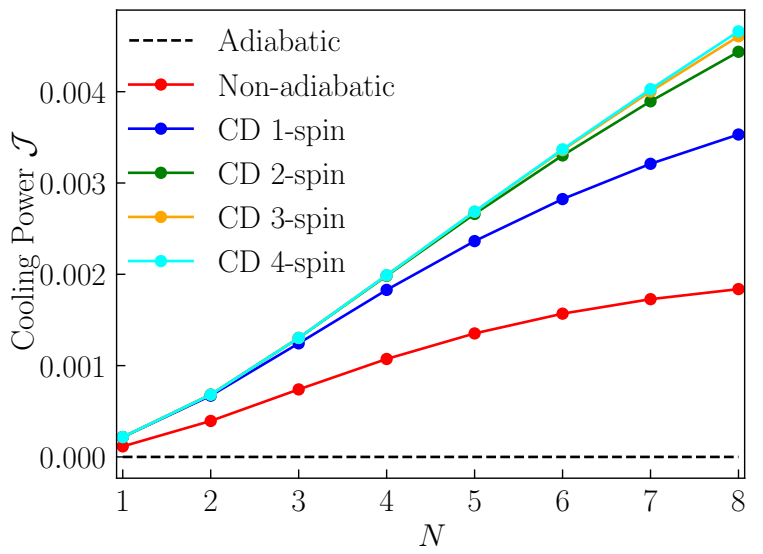

Figure 2: Cooling power $\mathcal{J}$ over system size $N$. Cooling power $\mathcal{J}$ of a quantum Otto cycle for isentropic stroke duration $\tau=\tau_{1}=\tau_{3} \approx 40$ for (i) non-adiabatic Hamiltonian $\left[H_{0}(t)\right.$, Eq. (3)] (red, bottom line) and (ii) STA Hamiltonian $\left[H_{\mathrm{STA}}^{*}(t)\right.$, Eq. (4)] for different $p$-spin CD terms $\left[H_{\mathrm{CD}}^{*}(t)\right.$, Eq. (5)] over different system sizes $N$. Parameters: Cold and hot bath at temperatures $T_{\mathrm{c}}=0.2$ and $T_{\mathrm{h}}=0.4$, respectively, and working parameters $\lambda_{\mathrm{c}}$ with $h_{j, \mathrm{i}}=0.2, b_{j, \mathrm{i}}=0$ and $J_{j k, \mathrm{i}}=0$ and $\lambda_{\mathrm{h}}$ with $h_{j, \mathrm{f}}=0, b_{j, \mathrm{f}}=0.5$ and $J_{j k, \mathrm{f}}=0.1$, magnetic field and interaction strengths, respectively. Duration of the thermalization strokes: $\tau_{2}=\tau_{4}=0.1$. Blackdashed line denotes cooling power at adiabatic limit where $\tau=\tau_{1}=\tau_{3} \rightarrow \infty$.

CD terms $\left[H_{\mathrm{CD}}^{*}\right.$, Eq. (5)] over different system sizes $N$ of spins in the WM for an isentropic stroke duration of $\tau=\tau_{1}=\tau_{3} \approx 40$. By applying $p$-spin CD terms, we can efficiently enhance the cooling power of our sped-up refrigerator by increasing the system size $N$ compared to the nonadiabatic counterpart. The relative enhancement in cooling power decreases the higher $p$ becomes. The major relative enhancement can be made by applying 1-spin or 2-spin CD terms. Including multi-spin terms (e.g. 3 and 4-body in the cases studied) only give a relatively slight improvement compared to 1-spin or 2-spin CD driving. We note, that there is a trade-off between enhanced cooling power with increasing $p$ and implementation complexity for an experiment. In the adiabatic limit (black-dashed line), the cooling power naturally converges to zero.

From a practical point of view, we deem it more favorable if the increased cooling power $\mathcal{J}_{\text {STA }}$ is due to the piston rather than exploiting the external control device. Consequently, we are interested in the work component $W_{\mathrm{CD}}^{1+3}$, Eq. (8). As shown in Appendix $\mathrm{C}$ the latter is zero if the applied CD Hamiltonian is exact.

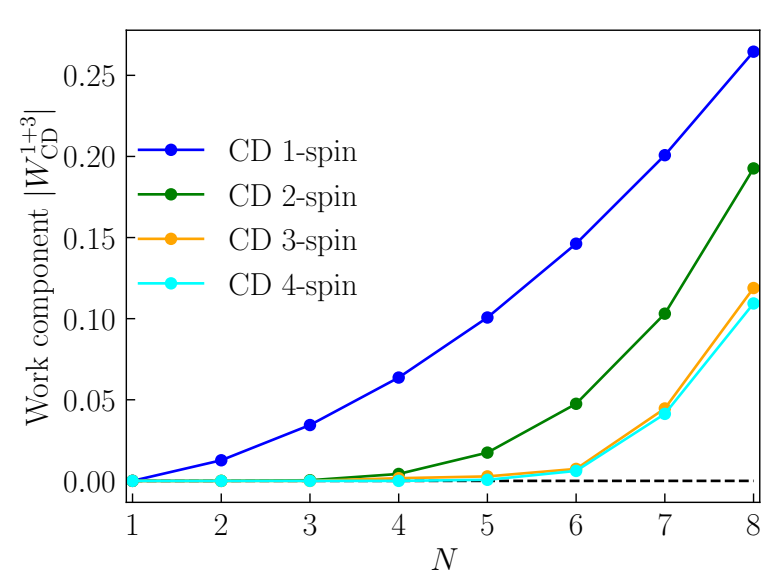

Figure 3: Work component $\left|W_{\mathrm{CD}}^{1+3}\right|$ over system size $\boldsymbol{N}$ for $\boldsymbol{p} \leq \mathbf{4}$. Absolute value of work component $W_{\mathrm{CD}}^{1+3}$ of the STA Hamiltonian $\left[H_{\mathrm{STA}}^{*}(t)\right.$, Eq. (4)] for different $p$-spin CD terms $\left[H_{\mathrm{CD}}^{*}(t)\right.$, Eq. (5)] over different system sizes $N$. Parameters: $\tau=\tau_{1}=\tau_{3}=1$. Other parameters as in Fig. 2.

Figure 3 depicts the absolute value of the work component $W_{\mathrm{CD}}^{1+3}$ stemming from the external control device during one cycle for different system sizes $N$ up to $p=4$. We see that $W_{\mathrm{CD}}^{1+3}$ is zero as long as $p>N$. Namely, the CD Hamiltonian must comprise all kinds of interactions up to order $N$, i.e., involving all the spins in the chain. By contrast, for $N>p$, i.e., more spins $N$ in the WM than order of interaction $p$ in $H_{\mathrm{CD}}^{*}(t)$, we see that the absolute value of $W_{\mathrm{CD}}^{1+3}$ adopts a non-zero value which, according to Appendix C, implies that the Hamiltonian $H_{\mathrm{CD}}^{*}(t)$ is not exact anymore. We therefore conclude, that including a CD Hamiltonian with all combinations up to $N$-body terms leads to an exact expression when the WM contains $N$ spins which is consistent with Refs. [42, 43]. These results encourage the goal to strive for an exact CD drive. For the latter, the external control device fully assists the piston that optimally "compresses" and "expands" the quantum WM.

\subsection{Dependence on cycle time}

We are further interested in the pumped heat $Q_{\mathrm{c}}$ per cycle and the corresponding $\mathrm{CoP}$ for different cycle durations $\tau$. Figure 4(a) shows the heat $Q_{\mathrm{c}}$ extracted from the cold reservoir over one cycle for a system size $N=6$. For the quantum Otto cycle with non-adiabatic Hamiltonian $H_{0}(t)$, we see that heat $Q_{\mathrm{c}}^{\text {na }}>0$ is pumped from the cold reservoir to the hot bath only for isentropic stroke 
(a)

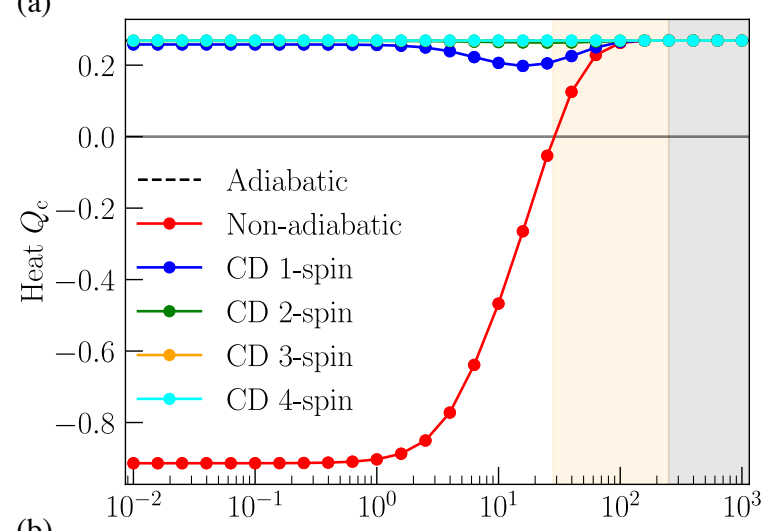

(b)

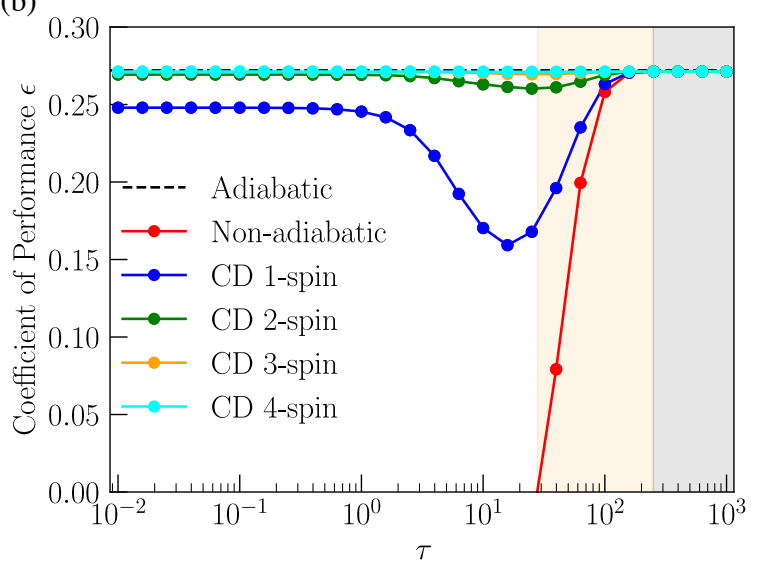

Figure 4: Pumped heat and coefficient of performance. (a) Pumped heat $Q_{\mathrm{c}}$ per cycle and (b) $\operatorname{CoP} \epsilon$ for (i) non-adiabatic Hamiltonian $\left[H_{0}(t)\right.$, Eq. (3)] and (ii) STA Hamiltonian $\left[H_{\mathrm{STA}}^{*}(t)\right.$, Eq. (4)] for different $p$-spin CD terms $\left[H_{\mathrm{CD}}^{*}(t), \mathrm{Eq} .(5)\right]$ over different isentropic stroke durations $\tau=\tau_{1}=\tau_{3}$ and system size $N=6$. Yellow-shaded area $(\tau \gtrsim 28)$ depicts the refrigerator regime where $Q_{\mathrm{c}}^{\text {na }}>0$ and the gray-shaded area $(\tau \geq 250)$ the adiabatic regime where $Q_{\mathrm{c}}^{\text {na }} \approx Q_{\mathrm{c}}^{\mathrm{ad}}$. Other parameters as in Fig. 2. durations $\tau=\tau_{1}=\tau_{3} \gtrsim 28$ (yellow-shaded area, refrigerator regime). For shorter isentropic stroke durations, the obtained states $\rho_{B}^{\prime}$ and $\rho_{D}^{\prime}$ at the end of each isentropic stroke (cf. Fig. 1) are so far away from the ideal adiabatic states $\rho_{B}$ and $\rho_{D}$ that the quantum Otto cycle ceases to describe a refrigerator. For large stroke durations, the refrigerator pumps the maximal possible cooling heat (gray-shaded area, adiabatic regime, $\tau \geq 250$ where $Q_{\mathrm{c}}^{\text {na }} \approx Q_{\mathrm{c}}^{\text {ad }}$ ).

By contrast, the sped-up cycle with Hamiltonian $H_{\mathrm{STA}}^{*}(t)$ including 1- up to 4-spin CD terms $H_{\mathrm{CD}}^{*}(t)$ even pumps heat from the cold reservoir in the quench limit $\tau_{1}, \tau_{3} \rightarrow 0$ where the cycle duration is dominated by thermalization. For increasing $p$, the cooling heat $Q_{\mathrm{c}}$ increases to its maximally possible value $Q_{\mathrm{c}}^{\text {ad }}$ for all durations studied. Note, however, that $p=2$ appears to be efficient even for the intermediate regime where $p=1$ does not yield optimal results. This is in accordance with Fig. 2. Note that for the adiabatic regime, the strength of the additional $H_{\mathrm{CD}}^{*}(t)$ converges to zero (as $\dot{\vartheta}(t) \propto 1 / \tau$, cf. Appendix A) and hence $Q_{\mathrm{c}} \rightarrow Q_{\mathrm{c}}^{\mathrm{ad}}$.

Figure 4(b) depicts the corresponding $\mathrm{CoP} \epsilon$, Eq. (10). For the sped-up cycle with $H_{\mathrm{STA}}^{*}(t)$, Eq. (4), we see that the higher $p$-spin terms we use for our CD Hamiltonian $H_{\mathrm{CD}}^{*}(t)$, Eq. (5), the larger the $\mathrm{CoP}$ becomes. Note that the $\mathrm{CoP}$ appears to be more sensitive to the value of $p$ than the pumped heat $Q_{\mathrm{c}}$. Namely, converging to the optimal $\mathrm{CoP} \epsilon^{\text {ad }}$, Eq. (2), generally requires a larger $p$ than for the optimal exctracted heat $Q_{\mathrm{c}}^{\text {ad }}$ which is due to a non-zero $W_{\mathrm{CD}}^{1+3}$.

\section{Discussion and Outlook}

In this work, we have presented a many-body quantum Otto refrigerator that efficiently operates at finite-time. The many-body quantum WM is modelled by an all-to-all connected Ising spin chain where the working parameter is parametrized via magnetic fields and interactions. In order to speed up the quantum Otto cycle, we apply an additional approximate multi-spin CD Hamiltonian. The latter outperforms its nonadiabatic and traditional 1-spin CD counterparts in pumped heat per cycle. The additional CD Hamiltonian contains $p$-spin terms with an odd number of $\sigma^{y}$ terms and $p \leq N$. For $p=N$, we obtain the exact CD Hamiltonian. We provide an 
analytical proof that the work component stemming from the external control device is zero if the CD Hamiltonian is exact. In this case, the external control device fully assists the piston to pump heat from a cold to a hot reservoir and acts as a catalyst. Note that while an exact CD protocol implies zero work contribution from the external control device over a cycle, the converse is not true. It is therefore not possible to find an exact CD Hamiltonian via minimizing this work component.

We numerically demonstrate an enhanced cooling power and coefficient of perfomance for the sped-up quantum Otto refrigerator with higher $p$-spin CD driving compared to its non-adiabatic and 1-spin counterparts. Furthermore, we show that increasing $p$ improves the scaling behaviour in cooling power with system size. For the Otto cycle with single-body quantum WM, i.e., described via the Landau-Zener model, we find an analytical expression for the CoP that only depends on the applied magnetic field strengths. The analytical and numerical performance analysis reveals that quantum Otto refrigerators using WM with even all-to-all connectivity can be scaled up efficiently by applying multi-spin CD protocols while increasing the number of spins in the WM.

We note that several cost identifiers for the additional CD Hamiltonian have been introduced [46, 47, 49, 51, 64-67]. We note, further, that these implementational costs are conceptually different to the operational costs described by the work $W_{\mathrm{CD}}^{1+3}$ stemming from the external control device [52]. In fact, in the case of exact CD driving we go on the optimal, i.e., adiabatic, path from points $A$ to $B$ and $C$ to $D$ in Fig. 1, respectively. Although the operational cost will then be zero, i.e., $W_{\mathrm{CD}}^{1+3}=0$, there will still be a cost of implementing the additional CD Hamiltonian. In Appendix E we have computed the strongly setup-dependent energetic cost of generating the additional magnetic fields of the CD Hamiltonian. However, although these energetic costs can be quantified, the actual implementation cost highly depends on the experimental setup and thus cannot be answered unambigously.

The results presented in this work disclose some important operational features. This work reveals a trade-off between experimental feasibility of the additionally applied CD terms and van- ishing work component stemming from the external control device. Namely, for the latter we need to apply non-local multi-spin CD terms (for example through additional laser fields) that are hard to implement in current experiments. On the other hand, applying only local 1-spin CD terms may result in enhanced cooling power compared to its non-adiabatic counterpart, yet under the price of extensive usage of the external control device rather than the piston. This work further shows that we can efficiently speed up the Otto cycle even for increasing system sizes by applying sufficiently well approximated multi-spin CD Hamiltonians. This work puts the results obtained in Ref. [52] into a wider context. In particular, this work significantly advances the ideas of the latter by providing a full assessment of experimental tuning parameters to consider and still being applicable for strongly site-dependent magnetic fields and interactions in a fully connected WM due to its increased complexity of external driving. While we have numerical evidence that 1-spin CD driving is just able to speed up the cycle for weakly coupled spins in the WM, e.g. with nearest-neighbor interaction, the higher-spin CD driving even generates cooling heat for strongly coupled and site-dependent magnetic fields and interactions for various all-to-all connected WM.

For future research, we intend to study the robustness of the applied CD protocols with respect to external noise and possible cooperative effects [68]. Furthermore, we aim at developing a many-body quantum refrigerator where work and heat exchanges occur simultaneously.

\section{Acknowledgements}

We thank Adolfo del Campo for valuable discussions. W.N. acknowledges support from an ESQ fellowship of the Austrian Academy of Sciences (ÖAW). V.M. acknowledges support from Science and Engineering Research Board (SERB) through Start-up Research Grant (Project No.SRG/2019/000411) and from Seed grant of IISER Berhampur. Work was supported by the Austrian Science Fund (FWF) through a START grant under Project No. Y1067-N27 and the SFB BeyondC Project No. F7108-N38, the Hauser-Raspe foundation, and the European Union's Horizon 2020 research and innovation program under grant agreement No. 817482. 
This material is based upon work supported by the Defense Advanced Research Projects Agency (DARPA) under Contract No. HR001120C0068. Any opinions, findings and conclusions or recommendations expressed in this material are those of the author(s) and do not necessarily reflect the views of DARPA.

\section{A Protocols for magnetic fields and in- teractions}

For the many-body quantum WM in the text, the explicit time dependence of the magnetic field and interaction strengths for the non-adiabatic $\left[H_{0}(t)\right.$, Eq. (3) $]$ and STA Hamiltonian $\left[H_{\text {STA }}^{*}(t)\right.$, Eq. (4)] read

$$
\begin{aligned}
h_{j}(t) & =h_{j, \mathrm{i}}+\left(h_{j, \mathrm{f}}-h_{j, \mathrm{i}}\right) \vartheta(t), \\
b_{j}(t) & =b_{j, \mathrm{i}}+\left(b_{j, \mathrm{f}}-b_{j, \mathrm{i}}\right) \vartheta(t), \\
J_{j k}(t) & =J_{j k, \mathrm{i}}+\left(J_{j k, \mathrm{f}}-J_{j k, \mathrm{i}}\right) \vartheta(t)
\end{aligned}
$$

where

$$
\vartheta(t):=\sin ^{2}\left[\frac{\pi}{2} \sin ^{2}\left(\frac{\pi t}{2 \tau_{l}}\right)\right]
$$

is the sweep function and $h_{j, \mathrm{i}}=h_{j}(t=0), b_{j, \mathrm{i}}=$ $b_{j}(t=0), J_{j k, \mathrm{i}}=J_{j k}(t=0)$ and $h_{j, \mathrm{f}}=h_{j}\left(t=\tau_{l}\right)$, $b_{j, \mathrm{f}}=b_{j}\left(t=\tau_{l}\right), J_{j k, \mathrm{f}}=J_{j k}\left(t=\tau_{l}\right)$ the initial and final values for each isentropic stroke $l \in\{1,3\}$ of duration $\tau_{l}$, respectively. Its derivative with respect to time reads

$$
\dot{\vartheta}(t)=\frac{\pi^{2}}{4 \tau_{l}} \sin \left(\frac{\pi}{\tau_{l}} t\right) \sin \left[\pi \sin ^{2}\left(\frac{\pi}{2 \tau_{l}} t\right)\right]
$$

and is applied to the CD Hamiltonian, Eq. (5).

\section{B Multi-spin CD driving}

For the CD Hamiltonian $H_{\mathrm{CD}}(t)$, Eq. (5) in the text, we apply an approximate adiabatic gauge potential $\mathcal{A}_{\vartheta}^{*}$. Here, we follow the variational method introduced in Ref. [42].

We make an ansatz $\mathcal{A}_{\vartheta}^{*}$ with $p$-spin Pauli matrices and an odd number of $\sigma^{y}$ terms for the adiabatic gauge potential and calculate the Hermitian operator $G_{\vartheta}\left(\mathcal{A}_{\vartheta}^{*}\right)=\partial_{\vartheta} H_{0}+i\left[\mathcal{A}_{\vartheta}^{*}, H_{0}\right]$. The goal is to minimize the operator distance $D^{2}\left(\mathcal{A}_{\vartheta}^{*}\right)=\operatorname{Tr}\left\{\left[G_{\vartheta}\left(\mathcal{A}_{\vartheta}\right)-G_{\vartheta}\left(\mathcal{A}_{\vartheta}^{*}\right)\right]^{2}\right\}$ between the exact, $\mathcal{A}_{\vartheta}$, and approximate, $\mathcal{A}_{\vartheta}^{*}$ adiabatic gauge potential. This minimization is equivalent to minimizing the action

$$
S\left(\mathcal{A}_{\vartheta}^{*}\right)=\operatorname{Tr}\left[G_{\vartheta}^{2}\left(\mathcal{A}_{\vartheta}^{*}\right)\right]
$$

with respect to its parameters in front of every Pauli matrix term, i.e., $\delta \mathcal{S}\left(\mathcal{A}_{\vartheta}^{*}\right) / \delta \mathcal{A}_{\vartheta}^{*}=0$.

As an example, for $p=1$, i.e., 1 -spin CD driving, we apply the ansatz $\mathcal{A}_{\vartheta}^{*}=\sum_{j=1}^{N} \alpha_{j} \sigma_{j}^{y}$ and calculate the Hermitian operator $G_{\vartheta}\left(\mathcal{A}_{\vartheta}^{*}\right)$ as well as the action $\mathcal{S}\left(\mathcal{A}_{\vartheta}^{*}\right)$. By minimizing the latter with respect to the coefficients $\alpha_{j}$, we find the optimal solution for each spin. For $p=2$, we apply the ansatz $\mathcal{A}_{\vartheta}^{*}=\sum_{j=1}^{N} \alpha_{j} \sigma_{j}^{y}+\sum_{k<j} \beta_{j k}\left(\sigma_{j}^{y} \sigma_{k}^{z}+\right.$ $\left.\sigma_{j}^{z} \sigma_{k}^{y}\right)+\gamma_{j k}\left(\sigma_{j}^{y} \sigma_{k}^{x}+\sigma_{j}^{x} \sigma_{k}^{y}\right)$ and solve the corresponding action with respect to all coefficients $\alpha_{j}, \beta_{j k}$ and $\gamma_{j k}$. With this variational method, we can also include multi-spin terms, potentially up to $N$-body terms $\sigma_{1}^{z} \cdots \sigma_{j}^{y} \cdots \sigma_{N}^{x}$. We solve the optimal form of each coefficient numerically.

\section{Proof of zero work component $W_{\mathrm{CD}}$ for exact $C D$ driving}

Here, we provide a detailed proof to the statement that the work contribution [52]

$$
W_{\mathrm{CD}}^{l}=\int_{0}^{\tau_{l}} \operatorname{Tr}\left[\rho(t) \dot{H}_{\mathrm{CD}}(t)\right] d t
$$

stemming from the external control device for each isentropic stroke $l \in\{1,3\}$ becomes zero if the additionally applied CD Hamiltonian $H_{\mathrm{CD}}^{*}(t)$ is exact. The latter reads [35]

$$
H_{\mathrm{CD}}(t)=i \hbar \sum_{n}|\dot{n}\rangle\langle n|-\langle n \mid \dot{n}\rangle| n\rangle\langle n|
$$

where $|n\rangle$ denotes the instantaneous eigenstate at time $t$. The density matrix $\rho(t)$ can be written as

$$
\rho(t)=\sum_{n} a_{n}|n\rangle\langle n|
$$

where $a_{n}$ is a coefficient whose sum fulfils the normalization relation. Here, we have used that under exact CD driving the density matrix remains diagonal in the instantaneous eigenbasis [69].

We now calculate the integral over the energy function

$$
f(t):=\operatorname{Tr}\left[\rho(t) \dot{H}_{\mathrm{CD}}(t)\right]
$$

for one isentropic stroke of duration $\tau$ [where $\dot{H}_{\mathrm{CD}}(t=0)=\dot{H}_{\mathrm{CD}}(t=\tau)=0$ ]. Employing the completeness condition $\sum_{n}|n\rangle\langle n|=\mathbb{1}$ of the eigenbasis, we receive the following relation

$$
\begin{aligned}
& \sum_{n} \partial_{t}^{2}(|n\rangle\langle n|)=\sum_{n}|\ddot{n}\rangle\langle n|+2| \dot{n}\rangle\langle\dot{n}|+| n\rangle\langle\ddot{n}|=0 \\
& \Rightarrow \sum_{n}|\dot{n}\rangle\langle\dot{n}|=-\frac{1}{2} \sum_{n}(|n\rangle\langle\ddot{n}|+| \ddot{n}\rangle\langle n|) . \quad(\mathrm{C} 5)
\end{aligned}
$$


On the other hand, the normalization condition $\langle n \mid n\rangle=1$ for each eigenstate separately leads to the following equations

$$
\begin{aligned}
& \partial_{t}(\langle n \mid n\rangle)=\langle\dot{n} \mid n\rangle+\langle n \mid \dot{n}\rangle=0, \\
& \langle\dot{n} \mid n\rangle=-\langle n \mid \dot{n}\rangle \\
& \Rightarrow 2\langle n \mid \dot{n}\rangle=\langle n \mid \dot{n}\rangle+\langle n \mid \dot{n}\rangle=\langle n \mid \dot{n}\rangle-\langle\dot{n} \mid n\rangle .
\end{aligned}
$$

Consequently, the time-derivative of the Hamiltonian $H_{\mathrm{CD}}(t)$ reads

$$
\begin{aligned}
\dot{H}_{\mathrm{CD}}(t) & =i \hbar \sum_{n}|\ddot{n}\rangle\langle n|+| \dot{n}\rangle\langle\dot{n}|-\partial_{t}(\langle n \mid \dot{n}\rangle|n\rangle\langle n|) \\
& \stackrel{(\mathrm{C} 5)}{=} i \hbar \sum_{n} \frac{1}{2}(|\ddot{n}\rangle\langle n|-| n\rangle\langle\ddot{n}|)-\partial_{t}(\langle n \mid \dot{n}\rangle)|n\rangle\langle n| \\
& -\langle n \mid \dot{n}\rangle(|\dot{n}\rangle\langle n|+| n\rangle\langle\dot{n}|) .
\end{aligned}
$$

The function $f(t)=\operatorname{Tr}\left[\rho(t) \dot{H}_{\mathrm{CD}}(t)\right]$, Eq. (C4), can consequently be rewritten (using $\operatorname{Tr}[\hat{H}]=$ $\left.\sum_{n}\langle n|\hat{H}| n\rangle\right)$ as

$$
\begin{aligned}
f(t) & =i \hbar \sum_{n} a_{n}\left[\frac{1}{2}(\langle n \mid \ddot{n}\rangle-\langle\ddot{n} \mid n\rangle)-\partial_{t}(\langle n \mid \dot{n}\rangle)-\langle n \mid \dot{n}\rangle(\langle n \mid \dot{n}\rangle+\langle\dot{n} \mid n\rangle)\right] \\
& \stackrel{(\text { C6 } 6)}{=} \frac{i \hbar}{2} \sum_{n} a_{n}\left[\langle n \mid \ddot{n}\rangle-\langle\ddot{n} \mid n\rangle-2 \partial_{t}(\langle n \mid \dot{n}\rangle)\right] \\
& \stackrel{(\text { C6 b })}{=} \frac{i \hbar}{2} \sum_{n} a_{n}\left[\langle n \mid \ddot{n}\rangle-\langle\ddot{n} \mid n\rangle-\partial_{t}(\langle n \mid \dot{n}\rangle-\langle\dot{n} \mid n\rangle)\right] \\
& =\frac{i \hbar}{2} \sum_{n} a_{n}[\langle n \mid \ddot{n}\rangle-\langle\ddot{n} \mid n\rangle-\langle\dot{n} \mid \dot{n}\rangle-\langle n \mid \ddot{n}\rangle+\langle\ddot{n} \mid n\rangle+\langle\dot{n} \mid \dot{n}\rangle] \\
& =0
\end{aligned}
$$

which proves the statement above.

\section{Single-body working medium}

In analogy to Ref. [49], we derive the CoP of the quantum refrigerator with a single-body WM.

The CoP, Eq. (10), in the text can be rewritten in terms of the energies $E_{i}$ with $i \in\{A, B, C, D\}$ at each point (cf. Fig. 1) of the cycle. It reads

$$
\epsilon_{\mathrm{LZ}}=\frac{E_{A}-E_{D}}{E_{B}-E_{A}+E_{D}-E_{C}}=\frac{1}{\frac{E_{B}-E_{C}}{E_{A}-E_{D}}-1}
$$

where the energies are

$$
\begin{aligned}
& E_{A}=-h_{\mathrm{x}, \mathrm{i}} \tanh \left(\frac{h_{\mathrm{x}, \mathrm{i}}}{T_{\mathrm{c}}}\right), \\
& E_{B}=-b_{\mathrm{z}, \mathrm{f}} \tanh \left(\frac{h_{\mathrm{x}, \mathrm{i}}}{T_{\mathrm{c}}}\right), \\
& E_{C}=-b_{\mathrm{z}, \mathrm{f}} \tanh \left(\frac{b_{\mathrm{z}, \mathrm{f}}}{T_{\mathrm{h}}}\right), \\
& E_{D}=-h_{\mathrm{x}, \mathrm{i}} \tanh \left(\frac{b_{\mathrm{z}, \mathrm{f}}}{T_{\mathrm{h}}}\right),
\end{aligned}
$$

due to the uniform scaling of the energy levels in this two-level system after each stroke. Inserting the latter, Eq. (D1) consequently reads

$$
\epsilon_{\mathrm{LZ}}=\frac{1}{\frac{b_{\mathrm{z}, \mathrm{f}}}{h_{\mathrm{x}, \mathrm{i}}}-1}
$$

which is equivalent to Eq. (11) from the text. 
The pumped heat

$Q_{\mathrm{c}}=E_{A}-E_{D}=h_{\mathrm{x}, \mathrm{i}}\left[\tanh \left(\frac{b_{\mathrm{z}, \mathrm{f}}}{T_{\mathrm{h}}}\right)-\tanh \left(\frac{h_{\mathrm{x}, \mathrm{i}}}{T_{\mathrm{c}}}\right)\right] \geq 0$

is positive for the Otto cycle to describe a refrigerator. From the latter and the fact that tanh is a monotonously increasing function, it follows that

$$
\frac{b_{\mathrm{z}, \mathrm{f}}}{h_{\mathrm{x}, \mathrm{i}}} \geq \frac{T_{\mathrm{h}}}{T_{\mathrm{c}}}
$$

Inserting this into Eq. (D3), we finally obtain

$$
\epsilon_{\mathrm{LZ}}=\frac{1}{\frac{b_{\mathrm{z}, \mathrm{f}}}{h_{\mathrm{x}, \mathrm{i}}}-1} \leq \frac{1}{\frac{T_{\mathrm{h}}}{T_{\mathrm{c}}}-1}=\frac{T_{\mathrm{c}}}{T_{\mathrm{h}}-T_{\mathrm{c}}}=\epsilon_{\mathrm{C}}
$$

with the Carnot $\mathrm{CoP} \epsilon_{\mathrm{C}}$ as the upper bound.

\section{E Implementation cost for multi-spin CD driving}

In this section we will consider the cost of implementing the additional Hamiltonian $H_{\mathrm{CD}}(t)$, Eq. (5) from the text. To this end, we will compute the energetic cost

$$
\left\langle H_{\mathrm{CD}}^{j}\right\rangle=\nu_{t, N} \int_{0}^{\tau} \operatorname{Tr}\left[H_{\mathrm{CD}}^{\dagger}(t) H_{\mathrm{CD}}(t)\right] d t
$$

of generating the additional magnetic fields of the CD Hamiltonian as defined in Ref. [66], for each isentropic stroke $j$ and squared Frobenius norm of the CD Hamiltonian, i.e. $\left\|H_{\mathrm{CD}}^{j}(t)\right\|^{2}$. Here $\nu_{t, N}$ is an experimental setup-dependent expression that can possibly be highly complicated to be evaluated.

Figure 5 depicts this energetic cost for different $p$-spin CD driving for (a) different sweep durations $1<\tau<10$ and system size $N=6$ and (b) different system sizes $N$ and sweep duration $\tau \approx 40$ with same parameters as in Fig. 2 . For panel (a) we have studied the sweep times $1<\tau<10$ as the strength of the additional magnetic fields are in the same order as the original magnetic fields and interactions, respectively, which we consider to be highly interesting for experimental realization.

We see that the energetic costs decrease (increase) exponentially for increasing sweep duration $\tau$ (system size $N$ ). Although the difference in energetic cost between each $p$-spin CD Hamiltonian are rather small, in particular in between (a)

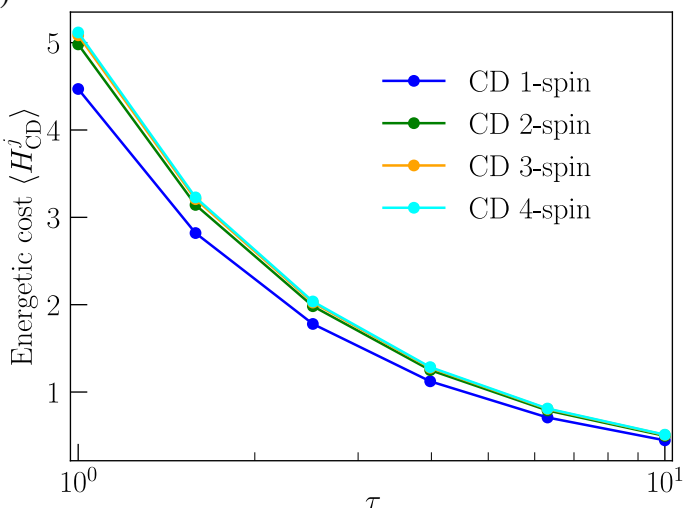

(b)

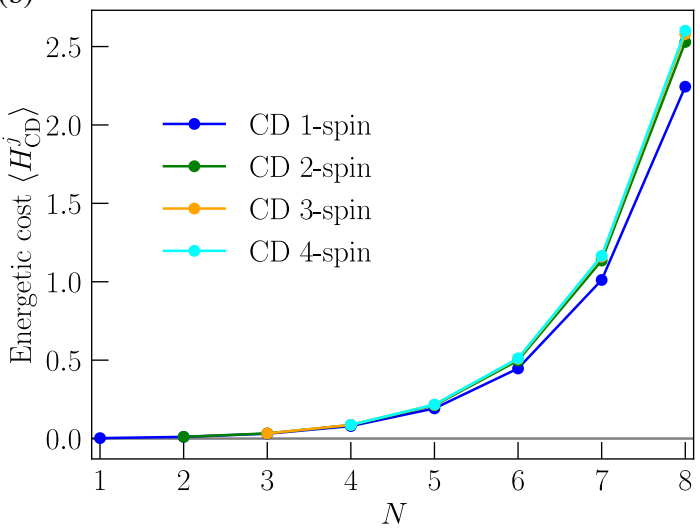

Figure 5: Energetic costs. Energetic cost $\left\langle H_{\mathrm{CD}}^{j}\right\rangle$, Eq. (E1), for implementation of the CD Hamiltonian $H_{\mathrm{CD}}^{j}(t)$, Eq. (5) from the text, during each isentropic stroke $j$ for (a) different sweep durations $1<\tau<10$ and system size $N=6$ and (b) different system sizes $N$ and sweep duration $\tau \approx 40$. Parameters: $\nu_{t, N}=0.01$ and other parameters as in Fig. 2.

2-spin and 4-spin CD driving, the actual implementation cost, i.e., implementing complicated non-local $N$-spin Hamiltonian rather than only local 1-spin terms, may differ considerably.

\section{References}

[1] Y. A. Çengel and M. A. Boles, Thermodynamics: An Engineering Approach, eighth ed. (McGraw-Hill Education, New York, 2015).

[2] R. Alicki, The quantum open system as a model of the heat engine, J. Phys. A 12, L103 (1979).

[3] R. Kosloff, A quantum mechanical open system as a model of a heat engine, J. Chem. Phys. 80, 1625 (1984).

[4] R. Kosloff, Quantum Thermodynamics: A Dynamical Viewpoint, Entropy 15, 2100 (2013). 
[5] D. Gelbwaser-Klimovsky, W. Niedenzu, and G. Kurizki, Thermodynamics of Quantum Systems Under Dynamical Control, Adv. At. Mol. Opt. Phys. 64, 329 (2015).

[6] S. Vinjanampathy and J. Anders, Quantum thermodynamics, Contemp. Phys. 57, 1 (2016).

[7] B. Karimi and J. P. Pekola, Otto refrigerator based on a superconducting qubit: Classical and quantum performance, Phys. Rev. B 94, 184503 (2016).

[8] R. Kosloff and Y. Rezek, The Quantum Harmonic Otto Cycle, Entropy 19 (2017), 10.3390/e19040136.

[9] F. Binder, L. A. Correa, C. Gogolin, J. Anders, and G. Adesso, eds., Thermodynamics in the Quantum Regime (Springer, Cham, 2019).

[10] S. Bhattacharjee and A. Dutta, Quantum thermal machines and batteries, (2020), arXiv:2008.07889 [quant-ph] .

[11] H. Bernien, S. Schwartz, A. Keesling, H. Levine, A. Omran, H. Pichler, S. Choi, A. S. Zibrov, M. Endres, M. Greiner, V. Vuletić, and M. D. Lukin, Probing manybody dynamics on a 51-atom quantum simulator, Nature 551, 579 (2017).

[12] J.-y. Choi, S. Hild, J. Zeiher, P. Schauß, A. Rubio-Abadal, T. Yefsah, V. Khemani, D. A. Huse, I. Bloch, and C. Gross, Exploring the many-body localization transition in two dimensions, Science 352, 1547 (2016).

[13] P. Bordia, H. Lüschen, S. Scherg, S. Gopalakrishnan, M. Knap, U. Schneider, and I. Bloch, Probing Slow Relaxation and Many-Body Localization in TwoDimensional Quasiperiodic Systems, Phys. Rev. X 7, 041047 (2017).

[14] J. V. Koski, V. F. Maisi, J. P. Pekola, and D. V. Averin, Experimental realization of a Szilard engine with a single electron, Proc. Natl. Acad. Sci. USA 111, 13786 (2014).

[15] J. Roßnagel, S. T. Dawkins, K. N. Tolazzi, O. Abah, E. Lutz, F. Schmidt-Kaler, and K. Singer, A single-atom heat engine, Science 352, 325 (2016).

[16] J. Klaers, S. Faelt, A. Imamoglu, and E. Togan, Squeezed Thermal Reservoirs as a Resource for a Nanomechanical Engine beyond the Carnot Limit, Phys. Rev. X 7, 031044 (2017).
[17] J. P. S. Peterson, T. B. Batalhão, M. Herrera, A. M. Souza, R. S. Sarthour, I. S. Oliveira, and R. M. Serra, Experimental Characterization of a Spin Quantum Heat Engine, Phys. Rev. Lett. 123, 240601 (2019).

[18] D. von Lindenfels, O. Gräb, C. T. Schmiegelow, V. Kaushal, J. Schulz, M. T. Mitchison, J. Goold, F. Schmidt-Kaler, and U. G. Poschinger, Spin Heat Engine Coupled to a Harmonic-Oscillator Flywheel, Phys. Rev. Lett. 123, 080602 (2019).

[19] J. Klatzow, J. N. Becker, P. M. Ledingham, C. Weinzetl, K. T. Kaczmarek, D. J. Saunders, J. Nunn, I. A. Walmsley, R. Uzdin, and E. Poem, Experimental Demonstration of Quantum Effects in the Operation of Microscopic Heat Engines, Phys. Rev. Lett. 122, 110601 (2019).

[20] Y. Rezek, P. Salamon, K. H. Hoffmann, and R. Kosloff, The quantum refrigerator: The quest for absolute zero, EPL 85, 30008 (2009).

[21] A. Levy and R. Kosloff, Quantum Absorption Refrigerator, Phys. Rev. Lett. 108, 070604 (2012).

[22] Y. Yuan, R. Wang, J. He, Y. Ma, and J. Wang, Coefficient of performance under maximum $\chi$ criterion in a two-level atomic system as a refrigerator, Phys. Rev. E 90, 052151 (2014).

[23] R. Long and W. Liu, Performance of quantum Otto refrigerators with squeezing, Phys. Rev. E 91, 062137 (2015).

[24] O. Abah and E. Lutz, Optimal performance of a quantum Otto refrigerator, EPL 113, 60002 (2016).

[25] W. Niedenzu, I. Mazets, G. Kurizki, and F. Jendrzejewski, Quantized refrigerator for an atomic cloud, Quantum 3, 155 (2019).

[26] H. B. Callen, Thermodynamics and an introduction to thermostatistics (Wiley, New York, 1985).

[27] E. Arimondo, P. R. Berman, and C. C. Lin, eds., Advances in Atomic, Molecular, and Optical Physics, Adv. At. Mol. Opt. Phys., Vol. 62 (Academic Press, 2013) pp. 117 - 169.

[28] A. del Campo and K. Sengupta, Controlling quantum critical dynamics of isolated systems, Eur Phys J Spec Top 224, 189 (2015).

[29] A. del Campo and K. Kim, Focus on Short- 
cuts to Adiabaticity, New J. Phys. 21, 050201 (2019).

[30] D. Guéry-Odelin, A. Ruschhaupt, A. Kiely, E. Torrontegui, S. Martínez-Garaot, and J. G. Muga, Shortcuts to adiabaticity: Concepts, methods, and applications, Rev. Mod. Phys. 91, 045001 (2019).

[31] R. Kosloff and T. Feldmann, Discrete fourstroke quantum heat engine exploring the origin of friction, Phys. Rev. E 65, 055102 (2002).

[32] T. Feldmann and R. Kosloff, Quantum fourstroke heat engine: Thermodynamic observables in a model with intrinsic friction, Phys. Rev. E 68, 016101 (2003).

[33] T. Feldmann and R. Kosloff, Quantum lubrication: Suppression of friction in a firstprinciples four-stroke heat engine, Phys. Rev. E 73, 025107 (2006).

[34] M. Demirplak and S. A. Rice, Adiabatic Population Transfer with Control Fields, J. Phys. Chem. A, J. Phys. Chem. A 107, 9937 (2003).

[35] K. Takahashi, Transitionless quantum driving for spin systems, Phys. Rev. E 87, 062117 (2013).

[36] X. Chen, A. Ruschhaupt, S. Schmidt, A. del Campo, D. Guéry-Odelin, and J. G. Muga, Fast Optimal Frictionless Atom Cooling in Harmonic Traps: Shortcut to Adiabaticity, Phys. Rev. Lett. 104, 063002 (2010).

[37] X. Chen, E. Torrontegui, and J. G. Muga, Lewis-Riesenfeld invariants and transitionless quantum driving, Phys. Rev. A 83, 062116 (2011).

[38] K. Takahashi, Transitionless quantum driving for spin systems, Phys. Rev. E 87, 062117 (2013).

[39] C. Jarzynski, Generating shortcuts to adiabaticity in quantum and classical dynamics, Phys. Rev. A 88, 040101 (2013).

[40] A. del Campo, Shortcuts to Adiabaticity by Counterdiabatic Driving, Phys. Rev. Lett. 111, 100502 (2013).

[41] B. Damski, Counterdiabatic driving of the quantum Ising model, J. Stat. Mech. Theory Exp. 2014, P12019 (2014).

[42] D. Sels and A. Polkovnikov, Minimizing irreversible losses in quantum systems by local counterdiabatic driving, Proc. Natl. Acad. Sci. USA 114, E3909 (2017).

[43] P. W. Claeys, M. Pandey, D. Sels, and
A. Polkovnikov, Floquet-Engineering Counterdiabatic Protocols in Quantum ManyBody Systems, Phys. Rev. Lett. 123, 090602 (2019).

[44] A. Hartmann and W. Lechner, Rapid counter-diabatic sweeps in lattice gauge adiabatic quantum computing, New J. Phys. 21, 043025 (2019).

[45] A. d. Campo, J. Goold, and M. Paternostro, More bang for your buck: Superadiabatic quantum engines, Sci. Rep. 4, 6208 EP (2014).

[46] O. Abah and E. Lutz, Performance of shortcut-to-adiabaticity quantum engines, Phys. Rev. E 98, 032121 (2018).

[47] O. Abah and M. Paternostro, Shortcut-toadiabaticity Otto engine: A twist to finitetime thermodynamics, Phys. Rev. E 99, 022110 (2019).

[48] L. Dupays, I. L. Egusquiza, A. del Campo, and A. Chenu, Superadiabatic thermalization of a quantum oscillator by engineered dephasing, Phys. Rev. Research 2, 033178 (2020).

[49] B. Çakmak and Ö. E. Müstecaplığlu, Spin quantum heat engines with shortcuts to adiabaticity, Phys. Rev. E 99, 032108 (2019).

[50] K. Funo, N. Lambert, B. Karimi, J. P. Pekola, Y. Masuyama, and F. Nori, Speeding up a quantum refrigerator via counterdiabatic driving, Phys. Rev. B 100, 035407 (2019).

[51] O. Abah, M. Paternostro, and E. Lutz, Shortcut-to-adiabaticity quantum Otto refrigerator, Phys. Rev. Research 2, 023120 (2020).

[52] A. Hartmann, V. Mukherjee, W. Niedenzu, and W. Lechner, Many-body quantum heat engines with shortcuts to adiabaticity, Phys. Rev. Research 2, 023145 (2020).

[53] J. I. Cirac and P. Zoller, Goals and opportunities in quantum simulation, Nat. Phys. 8, 264 (2012).

[54] I. M. Georgescu, S. Ashhab, and F. Nori, Quantum simulation, Rev. Mod. Phys. 86, 153 (2014).

[55] A. Lucas, Ising formulations of many NP problems, Front. Phys. 2, 5 (2014).

[56] T. Albash and D. A. Lidar, Adiabatic quantum computation, Rev. Mod. Phys. 90, 015002 (2018). 
[57] M. Kolodrubetz, D. Sels, P. Mehta, and A. Polkovnikov, Geometry and nonadiabatic response in quantum and classical systems, Phys. Rep. 697, 1 (2017).

[58] A. del Campo, M. M. Rams, and W. H. Zurek, Assisted Finite-Rate Adiabatic Passage Across a Quantum Critical Point: Exact Solution for the Quantum Ising Model, Phys. Rev. Lett. 109, 115703 (2012).

[59] S. Alipour, A. Chenu, A. T. Rezakhani, and A. del Campo, Shortcuts to Adiabaticity in Driven Open Quantum Systems: Balanced Gain and Loss and Non-Markovian Evolution, Quantum 4, 336 (2020).

[60] R. Dann, A. Tobalina, and R. Kosloff, Fast route to equilibration, Phys. Rev. A 101, 052102 (2020).

[61] R. Dann, A. Tobalina, and R. Kosloff, Shortcut to Equilibration of an Open Quantum System, Phys. Rev. Lett. 122, 250402 (2019).

[62] A. Das and V. Mukherjee, Quantumenhanced finite-time Otto cycle, Phys. Rev. Research 2, 033083 (2020).

[63] J. R. Johansson, P. D. Nation, and F. Nori, QuTiP 2: A Python framework for the dynamics of open quantum systems, Comput. Phys. Commun. 184, 1234 (2013).

[64] O. Abah and E. Lutz, Energy efficient quantum machines, EPL 118, 40005 (2017).

[65] S. Campbell and S. Deffner, Trade-Off Between Speed and Cost in Shortcuts to Adiabaticity, Phys. Rev. Lett. 118, 100601 (2017).

[66] Y. Zheng, S. Campbell, G. De Chiara, and D. Poletti, Cost of counterdiabatic driving and work output, Phys. Rev. A 94, 042132 (2016).

[67] A. Tobalina, I. Lizuain, and J. G. Muga, Vanishing efficiency of a speeded-up ionin-Paul-trap Otto engine, EPL 127, 20005 (2019).

[68] A. Manatuly, W. Niedenzu, R. RománAncheyta, B. Çakmak, Ö. E. Müstecaplığlu, and G. Kurizki, Collectively enhanced thermalization via multiqubit collisions, Phys. Rev. E 99, 042145 (2019).

[69] K. Funo, J.-N. Zhang, C. Chatou, K. Kim, M. Ueda, and A. del Campo, Universal Work Fluctuations During Shortcuts to Adiabaticity by Counterdiabatic Driving, Phys. Rev. Lett. 118, 100602 (2017). 